\title{
AN ORDINARY DIFFERENTIAL EQUATION ARISES IN PLANE CURVE EVOLUTION
}

\author{
YUNG-JEN LIN GUO
}

\begin{abstract}
Under some conditions on $f$, we prove that any nontrivial positive solution of the ordinary differential equation $u^{\prime \prime}+f(u)=0$ in $\mathbf{R}$ is periodic.
\end{abstract}

\section{Introduction}

In this paper, we consider the following ordinary differential equation

$$
u^{\prime \prime}+f(u)=0 \text { in } \mathbf{R} \text {. }
$$

We are interested in studying the existence of positive periodic solutions of the equation (1.1). Under some conditions on $f$, we prove that any positive solution of (1.1) is preiodic.

Throughout this paper, we assume that $f \in C^{1}(0, \infty), f^{\prime}>0$ in $(0, \infty), f(u)$ has a unique zero at $u=1$, and that

$$
\int_{0}^{1} f(s) d s=-\infty, \quad \int_{1}^{\infty} f(s) d s=\infty .
$$

Typical examples are

$$
f(s)=s-s^{-\beta}, \beta \geq 1 ; \quad f(s)=s-e^{1 / s} / e .
$$

The motivation for studying this problem is from the study the self-similar solutions of plane curve evolution equations, including curve shortening equation (cf. $[1,6,7])$ and affine curve evolution equation (cf. $[3,4,9]$ ).

Let $\Gamma(t)$ be a family of convex embedded curves in $\mathbf{R}^{2}$. Let $\kappa$ be the inward curvature of $\Gamma(t)$ and let $V$ be the inward velocity of $\Gamma(t)$ in the direction of its inward normal vector. In the generalized isotropic curvature flow equation, it is assumed that

$$
V=\kappa^{\alpha}, \quad 0<\alpha \leq 1 .
$$

Received March 30, 2000.

2000 Mathematics Subject Classification. 34A34, 34C25.

Key words and phrases. Periodic solution, ordinary differential equation. 
Let $\theta$ be the angle parameter. Then $V=V(\theta, t)$ and $\kappa=\kappa(\theta, t)$. From (1.3) and the equation

$$
V_{t}=\alpha V^{1+1 / \alpha}\left(V_{\theta \theta}+V\right)
$$

we obtain that $\kappa$ satisfies the evolution equation

$$
\kappa_{t}=\kappa^{2}\left[\left(\kappa^{\alpha}\right)_{\theta \theta}+\kappa^{\alpha}\right]
$$

Given any finite $T>0$. We consider the backward self-similar solution of (1.4) in the form

$$
\kappa(\theta, t)=(T-t)^{-1 /(\alpha+1)} \phi(\theta)
$$

Then $\phi$ satisfies

$$
\frac{1}{\alpha+1} \phi^{-1}=\left(\phi^{\alpha}\right)^{\prime \prime}+\phi^{\alpha}
$$

Set $u=(\alpha+1)^{\alpha /(\alpha+1)} \phi^{\alpha}$. Then $u$ satisfies the equation (1.1) with $f(u)=u-u^{-\beta}$, where $\beta=1 / \alpha \geq 1$.

It is trivial that $u \equiv 1$ is a solution of (1.1). We shall refer it as a trivial solution and only consider the positive classical solutions of (1.1).

We remark that a similar study of self-similar solutions for anisotropic curvature flow equations can be found in $[2,5,8]$. For more references for the affine plane curve evolution, we refer the readers to the paper of Angenent et al. [4] and references cited therein.

\section{Periodicity}

In this section, we shall prove that under the assumptions on $f$ stated in Section 1 any nontrivial solution of (1.1) is periodic. We shall only consider the nontrivial positive solutions.

Let

$$
F(u)=\int_{1}^{u} f(s) d s
$$

Note that $F(1)=0$ and $F(u)>0, \forall u>0, u \neq 1$. Also, it follows from (1.2) that

$$
F(u) \rightarrow \infty \text { as } u \rightarrow 0^{+} \text {and } u \rightarrow \infty
$$

Lemma 2.1. Any local solution $u$ of (1.1) can be continued to be a global solution in R. Moreover, there are two positive constants $m$ and $M$ such that $m \leq u \leq M$.

Proof. Multiplying the equation (1.1) by $u^{\prime}$, we obtain that the quantity

$$
\frac{1}{2}\left(u^{\prime}(x)\right)^{2}+F(u(x))
$$


is a constant, independent of $x$. Let $x_{0}$ be any point in the existence interval of $u$. Then we have

$$
F(u(x)) \leq \frac{1}{2}\left(u^{\prime}\left(x_{0}\right)\right)^{2}+F\left(u\left(x_{0}\right)\right)
$$

for any $x$ in the existence interval of $u$. It follows from (2.2) that there are two positive constants $m$ and $M$ such that

$$
m \leq u(x) \leq M
$$

From this estimate we conclude that any local solution can be continued to be a global solution in $\mathbf{R}$.

We say a function $u(x)$ is monotone ultimately at $\infty$ (or, $-\infty)$ if there is a constant $C$ such that $u$ is monotone for all $x \geq C$ (or, for all $x \leq C$ ).

Lemma 2.2. Suppose that $u$ is a solution of (1.1). If $u$ is monotone ultimately at $\infty$, then $u(x) \rightarrow 1$ as $x \rightarrow \infty$. Similar result holds for the case of $-\infty$.

Proof. Suppose that $u$ is monotone increasing ultimately at $\infty$. Then the limit

$$
l=\lim _{x \rightarrow \infty} u(x)
$$

exists and $l \in(0, \infty)$ by Lemma 2.1 .

Suppose that $u^{\prime}(x) \geq 0$ for all $x \geq C$ for some $C>0$. Since the integral

$$
\int_{C}^{\infty} u^{\prime}(x) d x
$$

is finite, there is a sequence $\left\{x_{n}\right\} \subset(C, \infty)$ such that $x_{n} \rightarrow \infty$ and $u^{\prime}\left(x_{n}\right) \rightarrow 0$.

If $l \neq 1$, then the integral

$$
\int_{C}^{x_{n}} \frac{f(u(x))}{x} d x
$$

is unbounded as $n \rightarrow \infty$. But, the integral

$$
\int_{C}^{x_{n}} \frac{u^{\prime \prime}(x)}{x} d x=\left[\frac{u^{\prime}\left(x_{n}\right)}{x_{n}}-\frac{u^{\prime}(C)}{C}\right]+\int_{C}^{x_{n}} \frac{u^{\prime}(x)}{x^{2}} d x
$$

is uniformly bounded for all $n$, a contradiction. This proves the lemma.

From Lemma 2.2, we see that any solution of (1.1) have at least one critical point. Note that any critical point is a maximum point if $u>1$ and is a minimum point if $u<1$. Also, there is no critical point with $u=1$, unless it is the trivial solution. Since the equation (1.1) is autonomous, we may assume without loss of generality that $u^{\prime}(0)=0$. Furthermore, since $u(-x)$ is also a solution if $u(x)$ is, it follows from the uniqueness theorem for the initial value problem of ordinary differential equation that any solution $u$ of (1.1) must be an even function.

Lemma 2.3. Any nontrivial positive solution $u$ of (1.1) must be oscilatory at both $\infty$ and $-\infty$. 
Proof. Otherwise, if $u$ is monotone ultimately at $\infty$, then $u(x) \rightarrow 1$ as $x \rightarrow \infty$. Recall that

$$
\frac{1}{2}\left(u^{\prime}(x)\right)^{2}+F(u(x))=F(u(0)) .
$$

It follows that

$$
\frac{1}{2}\left(u^{\prime}(x)\right)^{2} \rightarrow F(u(0))>0
$$

as $x \rightarrow \infty$, a contradiction.

We now state and prove the main theorem of this paper as follows.

Theorem 2.1. Any nontrivial positive solution of (1.1) is periodic.

Proof. Since any two adjacent critical points cannot be both maximum points, we may assume that $x=0$ is a minimum point. Let $\left\{x_{n}\right\}_{n \geq 0}$ be the increasing sequence of critical points of $u$ with $x_{0}=0$. Note that $x_{n}$ is a minimum point if $n$ is an even integer; and is a maximum point if $n$ is an odd integer. Let $\eta=u(0)$. Note that $\eta \in(0,1)$. From the assumptions on $f$ it follows that for each $\eta \in(0,1)$ there is a unique $\mu \in(1, \infty)$ such that $F(\mu)=F(\eta)$. Then by $(2.3)$

$$
u\left(x_{2 k}\right)=\eta, \quad u\left(x_{2 k-1}\right)=\mu, \quad \forall k \geq 1 .
$$

For any $k \geq 1$, since the function $u\left(x-x_{2 k}\right)$ is also a solution of (1.1) with the same initial value as $u(x)$ at $x=0$, it follows from the uniqueness of solutions of the initial value problem for ordinary differential equation that $u(x)=u\left(x-x_{2 k}\right)$. Hence we conclude that $u$ is periodic with minimal period $x_{2}$.

\section{An Example}

Let $u$ be a nontrivial solution of $(1.1)$ with $u^{\prime}(0)=0$ and $u(0)=\eta \in(0,1)$. Then by the result of Section 2 we see that $u$ is a periodic solution. Let $\omega$ be the minimal period of $u$. It is interesting to know how the period varies with the initial value.

Let $x_{1} \in(0, \omega)$ be the maximum point of $u$. Then $u\left(x_{1}\right)=\mu$. Recall that $F(\eta)=$ $F(\mu)$. From (2.3) it follows that

$$
\begin{aligned}
& \frac{u^{\prime}(x)}{\sqrt{2[F(\eta)-F(u(x))]}}=1,0<x<x_{1}, \\
& \frac{-u^{\prime}(x)}{\sqrt{2[F(\eta)-F(u(x))]}}=1, x_{1}<x<\omega .
\end{aligned}
$$

Hence by integrating the above two equations we obtain that

$$
\begin{aligned}
& \int_{\eta}^{\mu} \frac{d u}{\sqrt{2[F(\eta)-F(u)]}}=x_{1}, \\
& -\int_{\mu}^{\eta} \frac{d u}{\sqrt{2[F(\eta)-F(u)]}}=\omega-x_{1} .
\end{aligned}
$$


Hence $x_{1}=\omega / 2$ and so $x_{n}=n \omega / 2$. We conclude that

$$
\omega(\eta)=\sqrt{2} \int_{\eta}^{\mu} \frac{d u}{\sqrt{F(\eta)-F(u)}} .
$$

In general, it is very difficult to analyze the behavior of the improper integral (3.1). Some remarks are made on the case $f(u)=u-u^{-\beta}, \beta \geq 1$, as follows.

In [1], the authors proved with computer aided that $\omega(\eta)$ is monotone increasing in $\eta$ with range $(\pi, \sqrt{2} \pi)$ for $f(u)=u-u^{-1}$.

For $f(u)=u-u^{-3}$ (the affine curve evolution case), set $G(u)=u^{2}+u^{-2}$. Then we have $\mu=1 / \eta$ and

$$
\omega(\eta)=2 \int_{\eta}^{1 / \eta} \frac{d u}{\sqrt{G(\eta)-G(u)}} .
$$

Since by a change of variable $v=1 / u$

$$
\int_{1}^{1 / \eta} \frac{d u}{\sqrt{G(\eta)-G(u)}}=\int_{\eta}^{1} \frac{v^{-2} d v}{\sqrt{G(\eta)-G(v)}}
$$

we obtain

$$
\omega=2 \int_{\eta}^{1} \frac{\left(1+u^{-2}\right) d u}{\sqrt{G(\eta)-G(u)}}
$$

Introduce the variables

$$
\sigma^{2}=1-\frac{G(u)}{G(\eta)}, \quad a^{2}=1-\frac{2}{G(\eta)}
$$

We compute that

$$
\begin{aligned}
2 \sigma d \sigma & =-2 \frac{u-u^{-3}}{G(\eta)} d u, \\
\frac{1+u^{-2}}{u-u^{-3}} & =\frac{1}{u-u^{-1}}, \\
\left(u-u^{-1}\right)^{2} & =G(u)-2=\left(1-\sigma^{2}\right) G(\eta)-2=G(\eta)\left(a^{2}-\sigma^{2}\right) .
\end{aligned}
$$

Since $u \in(0,1)$, we have

$$
u-u^{-1}=-\sqrt{G(\eta)} \sqrt{a^{2}-\sigma^{2}} .
$$

It follows from (3.2) that

$$
\omega=2 \int_{0}^{a} \frac{d \sigma}{\sqrt{a^{2}-\sigma^{2}}}=\pi
$$

We conclude that any nontrivial solution of (1.1) with $f(u)=u-u^{-3}$ is periodic with minimal period $\pi$. It is independent of the initial value $\eta$. 
Indeed, this has also been observed in [3]. Since any solution $u$ satisfies the first integral

$$
u^{2}+\left(u^{\prime}\right)^{2}+u^{-2}=2 C,
$$

where $C$ is a positive constant. Hence $v=u^{2}$ satisfies the equation

$$
v^{\prime \prime}+4 v=4 C
$$

and we obtain the explicit solution formula

$$
v(x)=C+\sqrt{C^{2}-1} \sin \left[2\left(x-x_{0}\right)\right],
$$

for some $x_{0} \in \mathbf{R}$. Therefore, $u$ has period $\pi$.

\section{Acknowledgements}

The author would like to thank Professor J.-S. Guo for the interesting discussion and the referee for some useful comments. This work was partially supported by National Science Council of the Republic of China under the contract NSC 88-2115-M-003-002.

\section{References}

[1] U. Abresch and J. Langer, The normalized curve shortening flow and homothetic solutions, J. Diff. Geometry, 23(1986), 175-196.

[2] J. Ai, K. S. Chou and J. Wei, Self-similar solutions for the anisotropic affine curve shortening problem and a related nonlinear Hill's equation, Cal. Var. PDE, (to appear).

[3] B. Andrews, Contraction of convex hypersurfaces by their affine normal, J. Diff. Geometry, 43(1996), 207-230.

[4] S. Angenent, G. Sapiro and A. Tannenbaum, On the affine heat equation for non-convex curves, J. Amer. Math. Soc., 11(1998), 601-634.

[5] C. Dohmen, Y. Giga and N. Mizoguchi, Existence of selfsimilar shrinking curves for anisotropic curvature flow equations, Calc. Var., 4(1996), 103-119.

[6] M. Gage and R. Hamilton, The shrinking of convex plane curves by the heat equation, J. Diff. Geometry 23(1986), 69-96.

[7] M. Grayson, The heat equation shrinks embedded plane curves to round points, J. Diff. Geometry, 26(1987), 285-314.

[8] H. Matano and J. Wei, On anisotropic curvature flow equations, preprint.

[9] G. Sapiro and A. Tannenbaum, On affine plane curve evolution, J. Functional Analysis, 119(1994), 79-120.

Department of Mathematics, National Taiwan Normal University, 88, Sec. 4, Ting-Chou Road, Taipei 117, Taiwan

E-mail: yjguo@math.ntnu.edu.tw 